\title{
Fused Sensor Analysis and Advanced Control of Industrial Field Devices for Security
}

\author{
Cymbiote Multi-Source Sensor Fusion Platform
}

\author{
Theora Rice \\ theora.rice@pnnl.gov \\ Pacific Northwest National \\ Laboratory \\ Richland, WA
}

\author{
Garret Seppala \\ garret.seppala@pnnl.gov \\ Pacific Northwest National \\ Laboratory \\ Richland, WA
}

\author{
Thomas W. Edgar \\ thomas.edgar@pnnl.gov \\ Pacific Northwest National \\ Laboratory \\ Richland, WA
}

\author{
Daniel Cain \\ daniel.cain@pnnl.gov \\ Pacific Northwest National \\ Laboratory \\ Richland, WA
}

\author{
Eric Choi \\ eric.choi@pnnl.gov \\ Pacific Northwest National \\ Laboratory \\ Richland, WA
}

\begin{abstract}
Embedded field devices that sense and control physical processes in critical infrastructure are soft targets for cyber-attack because they lack the fundamental features for existing cyber security monitoring and control. In this paper we present a new platform to collect and fuse data across multiple cyber and physical streams from these field devices to enable the host based attack detection and security control capabilities that have become common within IT environments. Experiments of different attack behaviors are discussed and initial machine learning detection approach analysis is presented.
\end{abstract}

ACM Reference Format:

Theora Rice, Garret Seppala, Thomas W. Edgar, Daniel Cain, and Eric Choi. 2019. Fused Sensor Analysis and Advanced Control of Industrial Field Devices for Security : Cymbiote Multi-Source Sensor Fusion Platform. In Proceedings of Northwest Cybersecurity Symposium 2019 (Cybersecurity Symposium '19). ACM, New York, NY, USA, 8 pages. https://doi.org/10.1145/ 3332448.3332455

\section{INTRODUCTION}

It is now common knowledge that critical infrastructures and the sensors and controllers that operate within them are targets of cyber-attack. In the last decade, we have experienced the effects of malicious cyber behavior translate into cyber effects. Whether it is the destructive ramping of centrifuges[1], disruption of power[2], or the manipulation of safety systems to prevent dangerous failed process states [3]. Given the continued and increasing interest and attacks within critical infrastructure, it is more pressing than ever to find cyber security solutions for these systems.

Permission to make digital or hard copies of all or part of this work for personal or classroom use is granted without fee provided that copies are not made or distributed for profit or commercial advantage and that copies bear this notice and the full citation on the first page. Copyrights for components of this work owned by others than the author(s) must be honored. Abstracting with credit is permitted. To copy otherwise, or republish, to post on servers or to redistribute to lists, requires prior specific permission and/or a fee. Request permissions from permissions@acm.org.

Cybersecurity Symposium '19, April 08-10, 2019, Richland, WA

(c) 2019 Copyright held by the owner/author(s). Publication rights licensed to ACM

ACM ISBN $9781450366144 \ldots \$ 15.00$

https://doi.org/10.1145/3332448.3332455
Industrial and process control systems include many sensors and controllers distributed around some physical process such as the collection/generation and distribution of electric power, oil \& gas, water, as well as advanced manufacturing and chemical processing. Sensors and controllers are now generally embedded computational systems purpose-built for specific applications. These devices represent the touchpoint in the field between the cyber metaphysical space and the physical tangible space. As such, these field control devices (FCD) are often high risk cyber assets that are targets of cyber-attacks.

FCD are built for very specific use cases and are in place for a long period of time; on the order of 2-3 decades. As such, the computational and communication capabilities of field devices are generally built around older technology. Buying devices that still communicate over legacy Serial communication such as RS232/485 is still commonplace today. This has led to very limited or nonexistent cyber security functionality in FCDs. They generally lack cryptographic fundamentals, such as cryptographic processors, and common auditing logging. Since these are embedded platforms designed for specific tasks they generally do not allow additional monitoring software to be installed.

Given the critical nature of these systems to the smooth operation of our society it is necessary to develop cyber security solutions to protect these field devices from the threats they face. In this paper we present our research into developing a symbiotic cyber security platform to interface and interact with control system field devices. This device will fuse data from multiple data streams to improve context of attack detection and enable security controls within devices not designed for them. We contribute a design and prototype for a tool using commercial off-the-shelf (COTS) hardware, and present experiments to generate datasets for detection algorithms with initial analysis results.

\section{RELATED WORKS}

Defense in depth [4] is a foundational principle for securing cyber systems by layering defenses to reduce the risk of all security controls failing under an attack. Host based defenses have long been common security controls and sensors to provide one of the 
key layers of defense in IT networks. These go from the information theoretic models that drive information assurance and access controls within operating and file systems [5] to capabilities like antivirus[6], host intrusion detection systems (HIDS)[7], and sandboxing [8]. There are a myriad of previous work in host cyber security capabilities in information technology (IT) environments.

However, within the embedded and industrial control space this story is different. Because of the aging assets in operations, which were not designed with cyber security in mind, there is a lack of ability to upgrade and enhance existing equipment with traditional IT security controls. Therefore, research has gone into how to add security to systems where they currently are not possible.

Some lines of research are exploring modifications to device firmware to add protections to end devices. CodeSeal [9] utilized software trust anchors to embed cryptographic obfuscation to binaries to mitigate untrusted platforms. This approach requires a secondary oracle and a priori integration which limits effectiveness of securing existing hardware. The Symbiotic Embedded Machines (SEM) [10] injects sensoring and instruction set randomization into a firmware binary through inline function hooking and insertion into free memory locations. This methodology enables device agnostic modifications but it is risky without performance validation in operationally critical real-time safety systems and doesn't work when vendors follow best practice and sign their firmware. Finally, methods of using ladder logic additions[11] enable sensoring of field device performance and behavior. This is a very light impact method but it forces the sensors to operate within the untrusted space that is being targeted and manipulated by the attack and is potentially also effected.

Other research focuses on side channel, or sensoring of secondary measures of data, to detect abnormally behaving systems. Anomaly detection methodologies have been developed through the use of measuring acoustics [12], electromagnetic (EM) emissions [13] [14], and power consumption [15] [16]. Individually, these methods are all useful in detecting anomalous or rare behavior. However, without additional context and data they can't determine what caused the anomalous behavior lending toward relatively high false positive rates.

We propose that combining multiple methods will provide sufficient context to detect both the abnormality and the probable cause to improve the true-positive and decrease the false-positive detection rates. In the rest of this paper we describe a platform for collecting multiple data streams and initial analytics to fuse them together to provide detection of specific types of events that can enable operators and cyber security incident responders to optimally mitigate issues.

\section{APPROACH}

The ultimate goal of this effort is to identify whether an FCD has been effected, and if it has, identify what action caused the change in behavior. The context of this will inform what control response would be appropriate to apply at a device level in order to resume regular system activities. In order to accomplish this goal, however, we first had to identify how FCD features and data sources can be utilized to provide awareness and actuation. FCDs provide functionality and interfaces that have potential for collecting data about the operation of the system, and reconfigure the system into a more safe and secure state. In order to determine what is accessible, it is necessary to examine each category of FCD to determine what could serve as indicators of unusual behavior. To evaluate what controls can be enacted, the team looked at different commands and hardware interfaces that could be leveraged to change device settings and status.

When investigating these indicators and controls, we did not only investigate options that were readily available. Since it is unknown which indicators and controllers are valuable under various attack conditions, we wanted to find all possible device opportunities that could be leveraged for resiliency. The initial brainstorm included many ideas, ranging from obvious methods of checking logic configuration to more extreme ideas of installing weight scales within the racks to indicate physical tampering. Naturally, some of these are easier to implement than others. Empirical investigation, the start of which is presented in this paper, provides the means to determine the value of the various indicators and controls for detecting and responding to classes of cyber attacks.

In order to perform these experiments it was necessary to develop a platform implementing the sensors and actuators to interface with FCDs. We developed the Cymbiote prototype platform as an experimental tool for generating the data necessary to study the data density of various measurements and the effectiveness of controls in preventing and recovering from cyber attacks. In the end, the list of indicator and reaction ideas was shortened to those that were surmised to be the most relevant and could be measured accurately. These were then implemented in the Cymbiote platform, discussed later in this paper.

\subsection{Indicators and Reactions}

Among the potential indicators, six main categories were targeted: software, hardware, physical, side-channel, input/output (I/O), and contextual as seen in Table 1. Software describes items such as vendor-supplied tools and functionality, navigating the on-board file directories, and accessing different software commands for the individual on-board chipsets. Hardware refers to objects that can be derived or tapped from the hardware of the device itself. Physical indicators monitor the process, to alert when there is a change in behavior that is unexpected. Side-channel refers to indirect measurements taken of the FCD that may reflect the state of the device, such as its temperature, audio recordings, and movement. I/O refers to data points that present from information transmitting to, from, or within an FCD via the network, internal communications, or process communications. Finally, the contextual category addresses anything that is related to the regular operation of the system, such as employee schedules, HMI configuration changes, event reports, etc.

The FCD controls did not fall into categories like the indicators, but instead were rated on a scale of disruption to the device operation. Changing a value, uploading a new logic program, and changing IP addresses all represent low risk controls that will likely have minimal impact upon the device. Rebooting the device, reloading firmware, and/or turning off the device entirely, are on the opposite side of the scale, as they are necessary in more extreme cases. In this latter category, the potential damage of keeping the 
Table 1: List of possible indicators

\begin{tabular}{|c|c|}
\hline Indicator Category & Indicator Source \\
\hline Software & $\begin{array}{l}\text { Log files } \\
\text { Firmware revision } \\
\text { Firmware hash } \\
\text { Firmware image } \\
\text { Select memory regions } \\
\text { Configuration hash } \\
\text { Suspicious disassembled firmware } \\
\text { Execution time }\end{array}$ \\
\hline Hardware & $\begin{array}{l}\text { Device model } \\
\text { Serial number } \\
\text { Hardware options } \\
\text { Temperature } \\
\text { Jumper positions } \\
\text { Evidence of disassembly } \\
\text { Physically Uncloneable Functions } \\
\text { Tamper Indicator }\end{array}$ \\
\hline Physical & $\begin{array}{l}\text { Loss of measurement } \\
\text { Measurement jump } \\
\text { Measurement threshold }\end{array}$ \\
\hline Side-channel & $\begin{array}{l}\text { LED activity } \\
\text { Sound } \\
\text { EMF field } \\
\text { Power draw } \\
\text { Visual/Thermal } \\
\text { Timing/GPS } \\
\text { Weight }\end{array}$ \\
\hline $\mathrm{I} / \mathrm{O}$ & $\begin{array}{l}\text { SCADA I/O change } \\
\text { Communication failure } \\
\text { Protocol change } \\
\text { Malformed message } \\
\text { Latency/Jitter variations } \\
\text { Backplane communications } \\
\text { External communications }\end{array}$ \\
\hline Contextual & $\begin{array}{l}\text { Time-of-day } \\
\text { Configuration change } \\
\text { Event reports } \\
\text { Process heuristics/behavior } \\
\text { Human interaction/interference }\end{array}$ \\
\hline
\end{tabular}

device online must be greater than that of lack of availability. The full list of control options are listed in Table 2.

\subsection{Hardware Interface Investigation}

Once these categories were narrowed down, we investigated the possibility of collecting data from these sources or controlling the devices in the proposed ways. This required studying the possible connections and data sources available within an FCD. For this initial pilot we studied an Schweitzer Engineering Laboratories (SEL) 451 Protection, Automation, and Bay Control System. The process of communicating with different Integrated Circuits (ICs) in the SEL device requires several investigatory steps. First, we did a physical scan of the instrument and printed circuit boards
Table 2: List of possible controls

\begin{tabular}{l|l}
\hline Control Disruption Level & Control Objective \\
\hline \hline & Reflash firmware \\
& Modify firmware \\
& Shut down device \\
& Re-load program \\
& Load new program/logic \\
& Factory Reset \\
& Inject deception \\
& Remove power \\
& Reboot \\
& Remove module/add-on board \\
& Take control action \\
& Transfer to secondary system \\
& Wipe memory \\
& Wipe Disk/key \\
& Override internal bus comms \\
& Change operating mode \\
& Change jumper connections \\
\hline \multirow{5}{*}{ Unobtrusive } & Re-set time management system \\
& Change time input weighting \\
& Change measurement weighting \\
& Change Addressing \\
& Change interface used \\
& Change communication medium \\
& Enable/Disable services \\
& Modify tuning parameters \\
& Increase monitoring \\
& Collect Logs/Increase logins \\
& Physical/manual control \\
& Change control algorithm \\
& Change device account settings \\
\hline \multirow{5}{*}{ Mildly aggressive } & Deploy adaptive honeypot \\
& Network Containment \\
& Send field engineer \\
&
\end{tabular}

within the instrument to locate possible points of connectivity to access additional data streams and inject advanced control functions. We looked for Joint Test Action Group (JTAG) connections, Serial ports, and other points of inquiry such as Ethernet, Memory, and Daughter cards. We also noted the different ICs that have visible markings to find datasheets on pinouts, and programming types. We monitored these IOs using a logic analyzer, debugging tools, and JTAG analyzers. Most of our success has been using a Bus Blaster to deal with JTAG interactions.

We used a Bus Blaster to do JTAG Scans, and communicate with unlocked ICs. We were able to interact with several different ICs on the SEL device. Through this, we were able to gather a variety of information, typically the manufacturer and type of ICs. However, usually a locked IC won't provide much information on the locked JTAGs within the SEL 451. We were able interact and read information successfully from a MAX16048 EEPROM chip, which provides live data on voltages and settings for the printed circuit board. We are also able to send a reset signal that enabled the reboot control of the instrument. 
Table 3: ROHM Sensor Shield Sensors

\begin{tabular}{l|l}
\hline Sensor & Measurement Unit \\
\hline \hline Time & Seconds \\
Temperature & Degrees Celsius \\
UV & $\mathrm{mW} / \mathrm{cm} 2$ \\
Magnetic Fields & South/North Detect \\
Acceleration & $\mathrm{g}$ \\
Direction & $\mathrm{uT}$ \\
Barometric Pressure & $\mathrm{hPa}$ \\
Ambient Light & $\mathrm{Lx}$ \\
Proximity & $\mathrm{ADC}$ Count \\
Color & $\mathrm{ADC}$ Count \\
Orientation & Deg/Sec
\end{tabular}

\section{CYMBIOTE PLATFORM}

Development of a new prototype platform was necessary to collect the various data streams from FCDs. Primarily, the purpose of the device is to monitor individual FCDs, and therefore it needs to be fit within or alongside a given device in order to observe the host directly. Something that also was taken into consideration is that most FCDs will have at least slightly different structures and form factor, even within the same vendor series. From these constraints we designed our platform to be small, modular, and adaptable. We chose to name our device the Cymbiote, due to its cyber-driven symbiotic nature of integrating with FCDs.

\section{HARDWARE DESIGN}

An wealth of sensors exist already exist for various applications. As such we didn't desire to start from scratch. Instead, five hardware devices were leveraged as the basis to construct the Cymbiote: a Raspberry Pi 3, an Arduino Due, a Rohm Semiconductor (ROHM) sensor shield, a small electrical Ethernet tap, and the Adafruit AMG8833 IR Thermal Camera. Together these pieces were integrated to create our prototype Cymbiote data platform.

A Raspberry Pi 3 was chosen to run the bulk of the Cymbiote Software. Its responsibilities include collecting data from all attached sensors, performing local analysis of this data, sending alerts when appropriate to a centralized analysis server, and enacting control actions passed down based on the decision of that server. The Raspberry $\mathrm{Pi}$ was chosen due to its ease of use, wide availability, and small footprint. It has enough processing power to stream, store, and analyze data, without being overly expensive or difficult to configure.

The Arduino Due facilitates our use of the ROHM sensor shield, which is a COTS printed circuit board (PCB) configured with the sensors listed in Table 3. This specific shield was designed to work with the Arduino Uno by default. However, for our project we found that we needed more I/O pins in order to provide the flexibility for more sensors. The Arduino Due provides a similar configuration and programming platform to the Uno, with more capability for expansion. The ROHM sensor shield was chosen because its list of sensors measures most of the indicators from the environmental category of our approach, and allowed us to quickly progress to experimentation.

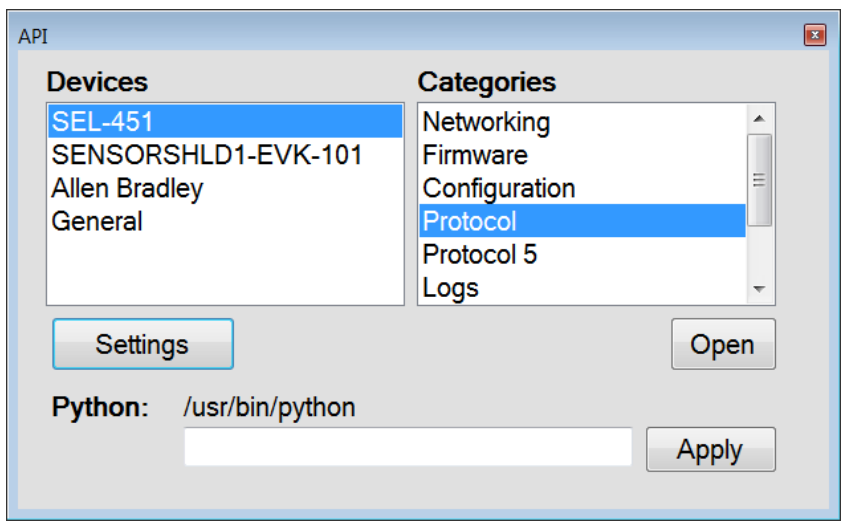

Figure 1: Graphical User Interface to Cymbiote API

To capture the network communications to and from the FCD, an electrical Ethernet tap was created. The tap duplicates the traffic going to and coming from the FCD for storage and analysis on the Raspberry Pi. Initially, the team had wanted to tap the Ethernet chip of the FCD directly. However it was found through implementation that this can often not be accomplished due to a lack of functionality on the chip.

The thermal camera was the last addition to this prototype setup, included based on the recommendation of a team at Idaho National Laboratory. This camera is meant to be pointed at the most heavily used chipsets within the FCD. It records a thermal map of the area it's pointed at and, thereby, record what components are being utilized the most and thus generating the most heat. The Adafruit AMG8833 IR thermal camera breakout was chosen because of its small size and ease of inclusion into the existing setup.

\subsection{Software Application Programming Interface (API)}

Cyber security operations need a way to communicate with the Cymbiote to monitor and control the FCD of which it is attached. An API was designed to enabled monitoring an FCDs many controls. The software runs on the raspberry PI component of the Cymbiote and consists of a restful web service running on the Python Flask micro web framework for remote accessibility. This gives analysts a way to interact with the FCDs and receive information. The API is designed to be generalized with few model specific extensions so that the Cymbiote can be connected to different models of FCD and still interface with a single central infrastructure. The controls that are available will change depending on which device the Cymbiote is connected to. Attempts to control unavailable functions results in failed response messages.

The core functionality of the API is built from scripts written in Python 2.7 which interact with the FCDs in order to read data, monitor settings, and configure the devices in a variety of way. Each script is generally responsible for a particular task per field control device, whether that task be to retrieve data or to change a setting. As an example, the API's functionality for the SEL-451 device described in our experimental section includes being able to 
monitor and change networking related settings. These settings include the IP address, MAC address, and default gateway. The range of functionality that the API provides highly depends on the FCD itself and which options it has available. The full scope for the SEL451 includes connection settings, device configuration, firmware versions, device logs, networking, passwords/authentication, and port protocol settings. Currently a Windows Forms GUI, written in C\#, provdes central collection and control.

Data analysis is intended to be run on the Cymbiote to detect anomalysis or suspicious events. Currently, the analysis applied to these readings is tailored to each FCD that the Cymbiote is installed in, with thresholds that dictate natural variability. If these thresholds are exceeded, it will trigger an alert. Upon detection of suspicious activity, the Cymbiote has facilities to generate syslog alerts and send it to remote collection servers. With these alerts operators or incident responders can decide what action the Cymbiote should take, if any, to mitigate a detected event. The available responses range from being able to revert the detected changes, power off/restart the device, change passwords/authentication settings, etc. The API is suited for both a Windows and a Linux based environment.

\section{EXPERIMENT}

Experiments were planned to study various analytic detection approaches for use in the Cymbiote and investigate the utility of indicators in identifying a specific event on the FCD. The hypothesis is that by evaluating data from multiple sources, the Cymbiote will have a more precise and higher detection accuracy than any individual data source. As an example, when a device with the Cymbiote attached is removed from the rack, multiple sensors will alert the Cymbiote logic that they have exceeded their thresholds. If these same sensors alert in a similar fashion every time the device is removed from the rack, but not during other events, then their values could be used to determine with high confidence that specific event is occurring.

\subsection{Threat Model}

The goal of this work is to enable advanced detection and defensive control of FCD devices from cyber attacks. The threat model defined in this section provides the adversarial perspective and those capabilities the Cymbiote is designed to protect against. Cyber-attacks against control system infrastructure could come from numerous sources, including malware attacking the system(s) via the Internet and/or malware that is introduced to the system via local means (e.g., a compromised memory stick). These can have numerous effects, including attacks against:

- Confidentiality: An attacker is able to extract market-valuable data. If inside information is acquired by a competitor or contractor, it decreases the negotiation power of the network owner.

- Availability: An attacker is able to disturb the flow of information and make it unavailable to the system operator, essentially creating a blind spot. In a distributed denial of service (DDoS) attack, an FCD is overloaded such that it cannot fulfill its regular duty.
Table 4: List of Experiment Trials

\begin{tabular}{l}
\hline Experiment Events \\
\hline Unplug Device \\
Unplug Ethernet cable \\
Plug Ethernet cable \\
Unplug front cable \\
Plug front cable \\
Enter password on face plate \\
Change device IP on face plate \\
Connect to a device with vendor software through face plate \\
Change device communications via Telnet \\
Connect to a device with vendor software over the network \\
Pull down device communication configuration over the network \\
Push device communication configuration over the network \\
Update configuration program with vendor software \\
Hit rip button \\
Open rack door \\
Close rack door \\
Unscrew device from rack \\
Remove device from rack \\
Select before operate control action \\
Direct operation control action \\
Upload firmware \\
Man in the middle
\end{tabular}

- Integrity: Even worse than an attack of availability, if an attacker causes nodes to communicate false information, either forcing the FCDs to improperly operate the process or causing the operator to question the process state and consequently make wrong decisions.

Both the compromise of availability and integrity have the potential for damage to FCDs as well as to the systems that depend on their correct operation. For example, if the power grid is targeted and subsequently fails, the ensuing blackout will have dramatic consequences for everyone.

We model the attacker with the following powers: Dolev-Yao [17] communication threat model: The adversary in this model can overhear, intercept, and synthesize any message and is only limited by the constraints of the cryptographic methods used. In other words: "the attacker carries the message." One addition to this model is that the adversary may have local access to the FCD to enact physical manipulation of the device. FCD are often in remote and unmanned facilities where an adversary could gain access to them. It is important to detect local actions to attack an FCD.

\subsection{Experiment Design}

To identify the different device actions that would drive our experiments, the team analyzed different cyber and physical methods through which a device could be compromised. Due to the wide variation between FCDs, these events needed to be generalized, so that they could apply to a wide range of devices. This process identified twenty two events that would need to be tested, so that the Cymbiote could collect data, and our team could analyze the results for correlations. These events are listed in Table 4. 
To compare against these events, 16 hours of data was collected as a baseline of operations. This baseline consisted of a single server routinely polling a single FCD for input status. This FCD was configured with default firmware. This baseline data was also used to configure the thresholds for the environmental sensors. Every individual device is going to have slightly different values for these sensors, based on both the device configuration (I.E. color, ambient light, etc.) and the environment the device is in (I.E. temperature, vibration, etc.) By evaluating how the sensors reported during this 16 hour span, the team was able to narrow down the reasonable amount of variation that each sensor experienced.

\subsection{Experiment Setup}

For our experiments, the team chose two devices to test: The SEL451 and the Allen Bradley ControlLogix. These devices represent different form factors, file structures, communications, and process purposes, which allowed us to exercise the flexibility of the Cymbiote device and experiment design.

For the SEL-451 tests, we connected the device to a Triangle Microworks OLE for Process Control (OPC) server, and configured a routine 2 second poll of the binary input status. Some of our tests we ran twice, once with the device resting on a table, once with the device screwed into a rack. This was initially to provide ease of access to the device as we figured out the optimum placement for the Cymbiote but also important to study the effect of FCD placement on the dependent variables. The Arduino, sensor shield, and thermal camera were placed inside the device, affixed to the lid, and pointed directly at the topmost board. A cable was threaded through the case, and to the Raspberry Pi that was affixed to the case exterior. A modified Ethernet cable was used to electrically tap the network communication such that copies of network packets are transmitted to both the Cymbiote and FCD.

To test the Cymbiote with the Allen-Bradley ControlLogix, we configured the FCD to perform routine actions within a building control system scenario. This included regular polling of BACnet values by a Johnson Controller NAE, as well as occasional change actions. The ControlLogix required a different attachment method for the Cymbiote. Instead of being one device with a single case, it has individual device modules that plug into a common backplane. Due to this difference, the team decided to focus on evaluating the BACnet Communication Interface Module by FieldServer technologies. To do this, we modified a blank module case to contain the Arduino, sensor shield, and thermal camera. We removed the side plate for the BACnet module so that the Cymbiote container could be placed next to it, and the environmental sensors could observe the module board. Once again, a cable ran from this device to the Raspberry Pi component affixed to the ControlLogix exterior. The network tap cable is placed to duplicate all network traffic to and from the BACnet module.

\section{INITIAL RESULTS}

As of the writing of this document, twenty out of the twenty two experiments in Table 4 have been run with the SEL-451. Each test was conducted in 10 trials, with all resulting data being recorded into PCAP, CSV, and raw text files. Although we have only begun to analyze the data, we have results from our initial investigation of the performance of various machine learning multi-class classification algorithms.

Of the resulting files from the experiments, we chose to focus on the environmental indicators in order to see if there was any correlation between the sensor readings and the event actions. Our initial analysis was to see how effective various multi-class classification algorithms were at identifying the scenarios used for each data set. For this work we selected the supervised learning approaches of K-Neighbors, Random Forest, Decision Tree, Gaussian Naïve Bayes, and Logistic Regression. We also used a random classification approach as a control case provide a minimum effectivness comparison floor.

\subsection{Data Pre-processing}

In order to run these algorithms it was necessary to pre-process the data. The data from the experiments was stored in a CSV format of sensor values sampled every half second. Each row of data needed to be collected into time windows of uniform size to be processed by the algorithms. The size of the time window selected could affect the performance of the classifier algorithms, therefore we tried each algorithm with window sizes every 5 seconds between 5 and 55 seconds. The environmental sensor data was read in matrix form and transposed before classification. The length of these tests varied due to some events being faster than others, which is not ideal as machine learning classifiers can only process sets of identicallysized vectors. Two actions were taken to overcome this. First, all test matrices smaller than the window were removed from training and validation, to avoid adding "fake" data that might bias the classifier. Second, all test matrices larger than the window were truncated to fit the data window size. The remaining truncated matrices were then flattened into vectors and used to train and validate the classifiers using the python-based scikit-learn machine [18] learning library. Each classifier used the default hyper-parameters.

Performance evaluation of each classifier was based on the F1 score, which is a well-established metric for machine learning algorithms. The F1 score is used because it considers accuracy and recall, which are both important in evaluating an algorithm's performance. It can be roughly interpreted as accuracy when comparing two algorithms, but note that the two are not logically equivalent. Ten F1 scores were collected for each classifier, with randomized training and testing sets for each iteration, and averaged. This procedure was repeated for window sizes from 5-55 seconds in 5 second steps. The results are shown in Table 5. Higher F1 scores represent better accuracy and recall of the classifier in classifying the events.

The random score represents the F1 score if the classifier chooses randomly from the available classification options. Note that it goes up with the window size. This is because the number of classification options decreases as the window size increases, as more shorter tests were removed from consideration. This raises the probability of randomly guessing correctly.

\subsection{Algorithm Performance}

Overall, the Random Forest algorithm produced the highest average F1 score, with Decision Tree being the next best. This makes sense, as most anomaly detection/identification problems intuitively lend themselves to a decision tree-based solution. Random Forest also 

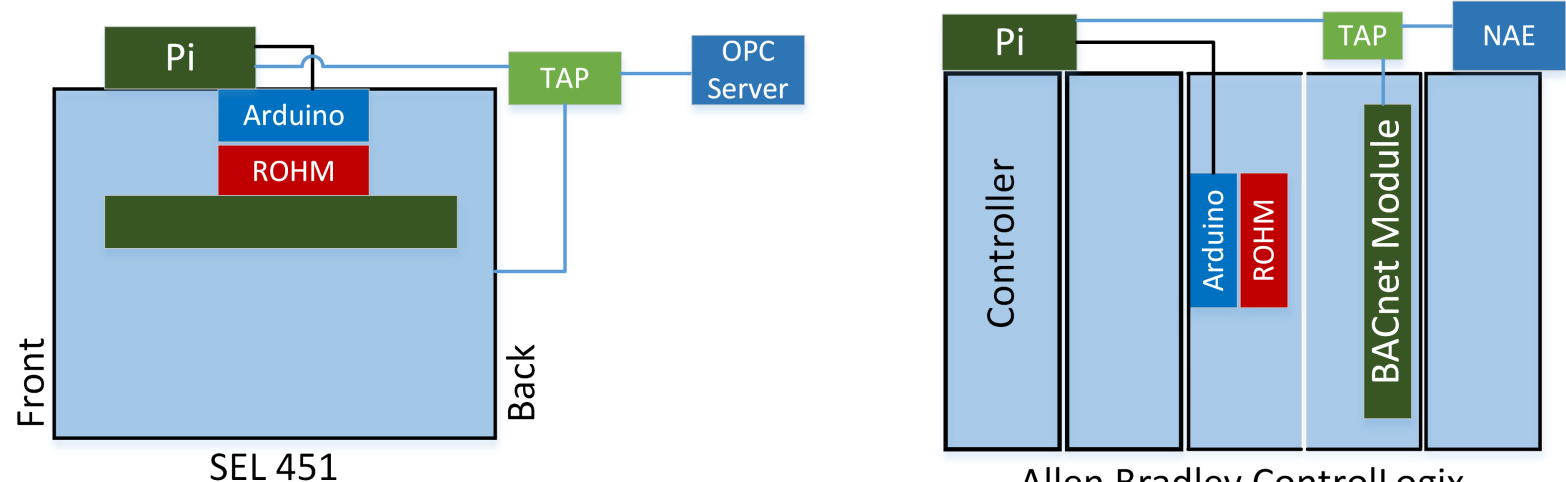

Allen Bradley ControlLogix

Figure 2: Experiment Setup for SEL-451 and ControlLogix

Table 5: Comparison of Machine Learning F1 Scores by Time Window Size

\begin{tabular}{|c|c|c|c|c|c|c|c|}
\hline $\begin{array}{c}\text { Window Size } \\
\text { (Seconds) }\end{array}$ & $\begin{array}{c}\text { Validation } \\
\text { Set Size }\end{array}$ & $\begin{array}{c}\text { Random } \\
\text { Average F1 }\end{array}$ & $\begin{array}{c}\text { K-Neighbors } \\
\text { Average F1 }\end{array}$ & $\begin{array}{c}\text { Random Forest } \\
\text { Average F1 }\end{array}$ & $\begin{array}{c}\text { Decision Tree } \\
\text { Average F1 }\end{array}$ & $\begin{array}{c}\text { Guassian } \\
\text { Naive Bayes } \\
\text { Average F1 }\end{array}$ & $\begin{array}{c}\text { Logistic } \\
\text { Regression } \\
\text { Average F1 }\end{array}$ \\
\hline \hline 5 & 30 & 0.0040 & 0.3977 & 0.7829 & 0.7408 & 0.6761 & 0.4843 \\
\hline 10 & 30 & 0.0029 & 0.5298 & 0.8097 & 0.7159 & 0.6262 & 0.5553 \\
\hline 15 & 30 & 0.0016 & 0.5026 & 0.7497 & 0.7197 & 0.6591 & 0.5806 \\
\hline 20 & 30 & 0.0119 & 0.4909 & 0.6970 & 0.6328 & 0.5973 & 0.5386 \\
\hline 25 & 30 & 0.0077 & 0.5038 & 0.6925 & 0.6181 & 0.5135 & 0.5267 \\
\hline 30 & 30 & 0.0024 & 0.4366 & 0.7499 & 0.6613 & 0.5636 & 0.5319 \\
\hline 35 & 30 & 0.0033 & 0.5566 & 0.7792 & 0.6993 & 0.5942 & 0.6040 \\
\hline 40 & 30 & 0.0198 & 0.5922 & 0.6809 & 0.6339 & 0.5362 & 0.6503 \\
\hline 45 & 30 & 0.0033 & 0.5731 & 0.6718 & 0.5800 & 0.5846 & 0.62717 \\
\hline 50 & 30 & 0.0251 & 0.5633 & 0.6758 & 0.6100 & 0.4575 & 0.6619 \\
\hline 55 & 30 & 0.0274 & 0.3310 & 0.4396 & 0.4366 & 0.3573 & 0.4561 \\
\hline
\end{tabular}

tends to perform better than Decision Tree, which also makes sense as the Random Forest algorithm creates many different decision trees before making a classification. Gaussian Naive Bayes also performs fairly well in some cases, although not as well as Decision Tree. K-Neighbors and Logistic Regression appear to be unfeasible for this problem.

The greatest drawback to these machine learning algorithms is that they all require input vectors of constant size. For a given data window size none of them may be trained on the tests that are shorter than that window. Overcoming this required attempting two methods of padding these shorter tests: zero-padding, and padding with data taken from a tamper-less baseline test. Each of these methods was determined to be unfeasible because the machine learning algorithms began to classify tests based on the padding length instead of the data itself. Future Cymbiote experiment data trials should be of constant length to eliminate this problem.

Another drawback to the machine learning approach in this scenario is the relatively small amount of data available to train with. Typical machine learning problems have dozens or hundreds of examples of each class to train and validate with, but currently each of the Cymbiote classes have ten or fewer examples. This severely limits the model's ability to generalize. Because of this, the machine learning algorithms were not able to indicate a predictable correlation between environmental sensors and events. However, the F1 scores have shown that there is likely some amount of correlation, just not strong enough to be actionable.

\section{CONCLUSIONS / FUTURE WORK}

In this paper, we discussed a framework and methodology for fusing multiple forms of data in a single platform to add enhanced detection and response capabilities to existing FCDs. We also presented some initial results of experiments to determine the effectiveness of using this platform to detect specific events that may represent an attack. While the results in this paper are preliminary, they show promise that utilizing multiple sources of data improve classification of events beyond abnormal and non-abnormal. In order to examine the utility of the indicator data, more work in identifying whether there is a correlation between the data and events must be done. The problems encountered with machine learning indicate that many more tests with more precise control of timing are needed to identify nuances in individual events. However, the data that has been collected to date can be used to determine whether 
there is any statistically significant difference between the data of an event and the baseline. This analysis is currently underway, and should provide some indication as to whether the indicators are providing any valuable data in given events.

The future of this project will include further testing and refinement of the Cymbiote, as well as further exploring what techniques can be applied to determine if there is strong correlation between events and indicator data. In the short term, the team will be running the same experiments conducted on the SEL-451 against the Allen Bradley ControlLogix. We are also working towards placing multiple Cymbiote devices within a more realistic environment to study the integration of the platform with existing tools and to demonstrate the capability under realistic conditions. This demonstration will also show the control aspect of the Cymbiote, as it will be subservient to a centralized analysis engine on the system. This system will be monitoring the entire environment, and will be passing control actions to the Cymbiote. Beyond these current efforts, the team wants to adapt the Cymbiote to many more devices, so that we can further evaluate the generalizability of the device and our experimental design.

\section{ACKNOWLEDGMENTS}

The research described in this paper is part of the Proactive, Adaptive Cybersecurity for Control Initiative at Pacific Northwest National Laboratory (PNNL). It was conducted under the Laboratory Directed Research and Development Program at PNNL, a multiprogram national laboratory operated by Battelle for the U.S. Department of Energy.

\section{REFERENCES}

[1] Ralph Langner. 2013. To Kill a Centrifuge A Technical Analysis of What Stuxnet's Creators Tried to Achieve. Technical report. The Langner Group, (November 2013).

[2] Anton Cherepanov. 2017. WIN32/Industroyer: A new threat for industrial control systems. Technical report. ESET, (June 2017).

[3] Blake Johnson, Dan Caban, Marina Krotofil, Dan Scali, Nathan Brubaker, and Christopher Glyer. 2017. Attackers Deploy New ICS Attack Framework "TRITON" and Cause Operational Disruption to Critical Infrastructure. Technical report. FireEye, (December 2017). https://www.fireeye.com/blog/ threat-research/2017/12/attackers-deploy-new-ics-attackframework-triton.html.

[4] Michael Coole. 2012. Defence in depth, protection in depth and security in depth: a comparative analysis towards a common usage language. In The Proceedings of the 5th Australian Security and Intelligence Conference, At Perth, Western Australia. (December 2012).

[5] E. Sahafizadeh and S. Parsa. 2010. Survey on access control models. In 2010 2nd International Conference on Future Computer and Communication. Volume 1. (May 2010), V1-1-V1-3. DOI: 10.1109/ICFCC.2010.5497850.

[6] Nwokedi C. Idika. 2007. A Survey of Malware Detection Techniques. Technical report. Purdue University.

[7] Zahra Bazrafshan, Hashem Hashemi, Seyed Mehdi Hazrati Fard, and Ali Hamzeh. 2013. A survey on heuristic malware detection techniques. In IKT 2013 - 2013 5th Conference on Information and Knowledge Technology. (May 2013), 113-120. ISBN: 978-1-4673-6489-8. DOI: 10.1109/IKT.2013.6620049.

[8] Michael Maass, Adam Sales, Benjamin Chung, and Joshua Sunshine. 2016. A systematic analysis of the science of sandboxing. Peerf Computer Science, 2, (January 2016), e43. DOI: 10.7717/peerj-cs.43.

[9] Adrian Chavez and John Hector Solis. 2015. Code seal v. 2.0, version 00. (April 2015).

[10] Ang Cui and Salvatore J. Stolfo. 2011. Defending embedded systems with software symbiotes. In Proceedings of the 14th International Conference on Recent Advances in Intrusion Detection (RAID'11). Springer-Verlag, Menlo Park, CA, 358-377. ISBN: 978-3-642-23643-3. DOI: 10.1007/978-3-642-23644-0_19. http://dx.doi.org/10.1007/978-3-642-23644-0_19.

[11] H. Yang, L. Cheng, and M. C. Chuah. 2018. Detecting payload attacks on programmable logic controllers (plcs). In 2018 IEEE Conference on Communications and Network Security (CNS). (May 2018), 1-9. DOI: 10.1109/CNS.2018.8433146.

[12] Wenming Cao, Erqian Tang, and Guanzheng Tan. 2015. Realtime monitoring of unmanned substation based on audio recognition. In 2015 3rd International Conference on Machinery, Materials and Information Technology Applications. (January 2015). DOI: 10.2991/icmmita-15.2015.272.

[13] Pol Van Aubel, Kostas Papagiannopoulos, Lukasz Chmielewski, and Christian Doerr. 2017. Side-channel based intrusion detection for industrial control systems. CRITIS, (December 2017).

[14] Ang Cui Nathaniel Boggs Jimmy C. Chau. 2018. Utilizing electromagnetic emanations for out-of-band detection of unknown attack code in a programmable logic controller. In Proceedings of SPIE. Volume 10630, 10630 - 10630 -16. DOI: 10.1117/12.2304465. https://doi.org/10.1117/12.2304465.

[15] Xiao Wang, Quan Zhou, Jacob Harer, Gavin Brown, Shangran Qiu, Zhi Dou, John Wang, Alan Hinton, Carlos Aguayo Gonzalez, and Peter Chin. 2018. Deep learning-based classification and anomaly detection of side-channel signals. Proc.SPIE, 10630, 10630 - 10630 -8. DOI: 10.1117/12.2311329. https: //doi.org/10.1117/12.2311329.

[16] Yu-jun Xiao, Wen-yuan Xu, Zhenhua Jia, Zhuo-ran Ma, and Dong-Lian Qi. 2017. Nipad: a non-invasive power-based anomaly detection scheme for programmable logic controllers. Frontiers of Information Technology \& Electronic Engineering, 18, (April 2017), 519-534. DOI: 10.1631/FITEE.1601540.

[17] D. Dolev and A. Yao. 1983. On the security of public key protocols. IEEE Transactions on Information Theory, 29, 2, (March 1983), 198-208. ISSN: 0018-9448. DOI: 10.1109/TIT. 1983.1056650.

[18] F. Pedregosa, G. Varoquaux, A. Gramfort, V. Michel, B. Thirion, O. Grisel, M. Blondel, P. Prettenhofer, R. Weiss, V. Dubourg, J. Vanderplas, A. Passos, D. Cournapeau, M. Brucher, M. Perrot, and E. Duchesnay. 2011. Scikit-learn: machine learning in Python. Journal of Machine Learning Research, 12, 28252830. 